\title{
PERSPECTIVE
}

\section{Complications of the Type 2 Diabetes Mellitus}

\author{
Paraskevi Farmaki ${ }^{1}$, Christos Damaskos ${ }^{2}$, Nikolaos Garmpis ${ }^{2}$, Anna Garmpi ${ }^{3}$, Spyridon Savvanis ${ }^{4}$ \\ and Evangelos Diamantis, ${ }^{5, *}$
}

\author{
${ }^{I}$ First Department of Pediatrics, Aghia Sophia Children's Hospital, Athens, Greece; ${ }^{2}$ Second Department of Propedeutic \\ Surgery, Laiko General Hospital, Medical School, National and Kapodistrian University of Athens, Athens, Greece; \\ ${ }_{3}^{3}$ Internal Medicine Department, Laiko General Hospital, Medical School, National and Kapodistrian University of Ath- \\ ens, Athens, Greece; ${ }^{4}$ Department of Internal Medicine, General Hospital of Athens "Elpis", Athens, Greece; ${ }^{5}$ Health \\ Center Peristeriou, Athens, Greece
}

\section{INTRODUCTION}

In type 2 diabetes mellitus, there is an inadequate production of insulin or the insulin produced does not meet its purpose of introducing glucose into the cells, thus depriving them of energy. This condition is called "insulin resistance" because while there is insulin, it cannot introduce glucose into the cells and thus there is an accumulation of glucose in the blood. This is also the main disorder that occurs in the early stages of type 2 diabetes.

However, individuals can regulate the glucose produced by doing more exercise, reducing their weight and avoiding high carbohydrate foods. Insulin resistance, of course, can be continued, so patients should continue and adjust properly by means of special training, physical exercise, diet, control of their body weight, and an addition of medication (antidiabetic pills) [1]. Over time, if pancreatic cells become increasingly inactive, then patients switch to insulin therapy to properly regulate glucose and insulin levels.

Type 2 diabetes appears much later in age than Type 1 diabetes and is the most common type of diabetes. It is also called as non-insulin-dependent, since it is not treated exclusively by the use of insulin, but mainly with pills [2].

Type 2 diabetes mellitus, usually shows (because there may not be any symptom) the following symptoms: frequent urination, especially in the evening (nocturia) - polyuria, polydipsia, polyphagia and intense hunger, weight loss, weakness / tiredness, lack of interest and concentration, vomiting and stomach pain, blurred vision, common infections and inflammation and wounds that are slow to heal and tingling at the extremities.

\section{COMPLICATIONS OF TYPE 2 DIABETES MELLITUS}

The complications of diabetes mellitus are distinguished in terms of being acute and chronic.

\section{THE ACUTE COMPLICATIONS OF TYPE 2 DIABETES MELLITUS}

\subsection{Diabetic Ketoacidosis and Diabetic Coma}

Diabetic ketoacidosis is a dangerous complication. It is considered an urgent event and requires the immediate transfer of the patient to the hospital. The patient has signs of dehydration, Kussmaul breath (deep, prolonged and sigh breathing) and acetone aspiration. Often, these signs are accompanied by diffuse abdominal pain. The level of consciousness is not affected initially, but then there may be a gradual decline in consciousness leading to sleepiness, lethargy and ultimately coma. In more severe cases, hypotension and circulatory shock are observed. With appropriate and timely treatment, diabetic ketoacidosis is a completely reversible condition [3].

\subsection{Hypoglycemia}

Hypoglycemia occurs when blood sugar is very low and is a major complication of diabetes treatment. It may be caused by an incorrect dose of insulin (increased dose), intense exercise, or reduced intake of food or carbohydrate. The patient has irritability and increased sweating while there may be disorders of the level of consciousness, loss of consciousness and / or coma. Immediate ingestion of glucose by mouth (sweets, sugary soft drinks, etc.) or, if the patient is unconscious, intravenous glucose administration is required. The symptoms of hypoglycemia include: increased sweating, blurred vision, trembling, headache or dizziness, skin paleness, irritability, tears leakage, convulsions, distraction of attention (absent-mindedness), disturbances of perception, clumsy moves, feeling tingled around the mouth and intense desire to eat.

"Address correspondence to this author at the Christos Ladas 43 street, Peristeri, Greece; E-mail: vaggelisd01@gmail.com 


\subsection{Hyperglycemia}

Hyperglycemia is called the condition in which blood sugar levels are too high. Hyperglycemia should be treated as it is the primary cause of serious and life-threatening complications in diabetes. It appears when there is no or insufficient insulin in the blood or insulin that is not working properly [4]. More often a person with diabetes mellitus develops hyperglycemia if he or she misses his or her medication or misses one or more doses. Other causes that can lead to hyperglycemia include eating sweets without proper treatment regimens or a possible infection. It is dealt with redesigning the already used therapeutic regimen, with a balanced diet and exercise [1].

\section{THE CHRONIC COMPLICATIONS OF TYPE 2 DIABETES MELLITUS}

Diabetes mellitus type 2 is a disease that adversely affects the functioning of almost every organ of the human body in the long run. Thus, the biggest problem for diabetic patients is the long-term complications that accompany the disease. The most common of these are [2]:

(i) Macroangiopathy: It concerns serious heart and vascular lesions that lead to hypertension, artery narrowing, coronary artery disease, strokes and erectile dysfunction in men.

(ii) Diabetic retinopathy: It causes a serious deterioration of vision mainly due to damage to the vessels of the eye. It is the most common cause of blindness in the Western world.

(iii) Diabetic nephropathy: which may result in renal insufficiency.

(iv) Diabetic neuropathy: It occurs with sensory disturbances, muscle atrophy, walking difficulty, injuries with wound formation and intense pain at the lower extremities. It is also responsible for tachycardia, orthostatic hypotension, urinary incontinence, indigestion, nausea, diarrhea and / or constipation.

(v) Diabetic foot: By this, we refer to the lesions observed on the diabetics at the region by the knees and below and are related to pain, sensory disorder, skin dryness, development of calluses, wounds and ulcers, often complicated by severe local infections and leading to the development of gangrene with amputation of the fingers.

(vi) Vulnerability to infections, myopathy, osteoporosis, arthropathies and liver damage are additional conditions often associated with diabetes mellitus.

\section{HEART DISEASE AND DIABETES MELLITUS}

Almost all morbidity in type 2 diabetes mellitus has to do with cardiovascular diseases such as [2]:

- Coronary disease,

- Congestive heart failure;

- Arterial hypertension.

Type 2 diabetes mellitus disorders in heart function include [2]:

- Disturbances in the intensity of cardiac contraction,

- Disorders in the genesis and transmission of the stimulus resulting in changes in the heart rate,

- Disturbances in the heart muscle perfusion.

Type 2 diabetes mellitus has been characterized as equivalent to coronary artery disease, while many patients with confirmed coronary artery disease suffer from diabetes mellitus or its precursor forms. $20-30 \%$ of patients with acute coronary syndromes have type 2 diabetes mellitus, while $40 \%$ of them have impaired glucose tolerance [5]. There is evidence that rates of mortality after acute myocardial infarction are twice as high for patients with diabetes as compared to patients without diabetes [5].

Therefore, cardiovascular mortality is increased by 2 to 8 times in patients with type 2 diabetes mellitus, while $75 \%$ of deaths in these patients are attributable to the underlying coronary artery disease [6]. There is evidence to suggest that in addition to coronary artery disease, type 2 diabetes mellitus is an important risk factor for arrhythmias.

The risk for cardiovascular autonomic neuropathy depends on the duration of diabetes and the degree of glycemic control and tends to co-exist along with the development of complications of other target organs such as retinopathy, nephropathy and angiopathy [7]. It is estimated that $20 \%$ of asymptomatic patients with FD have approximately cardiovascular autonomic neuropathy

Cardiovascular autonomic neuropathy may also reduce the subjective perception of myocardial ischemia. Thus, patients with diabetes have less ischemic symptoms, such as angina compared to patients without diabetes [8].

In addition, tachycardia at rest, an early manifestation of the parasympathetic system disorder in cardiovascular autonomic neuropathy, increases myocardial oxygen needs and may bring the diabetic patient closer to the ischemic threshold [6]. Addi- 
tionally, cardiovascular autonomic neuropathy may cause coronary disorder vasomotor regulation, thereby disrupting the balance between myocardial delivery and demand [6].

There is evidence to suggest that QT interval prolongation in patients with diabetes is correlated with the degree of autonomic neuropathy and is an important predisposing factor for ventricular arrhythmogenesis and sudden cardiac death [7, 8].

Experimental data suggest that disturbance of the sympathetic system's function in diabetes increases the incidence of atrial fibrillation [9]. Furthermore, studies have shown that delayed intravaginal treatment and vaginal fibrosis development are important predisposing factors for atrial tachyarrhythmia diabetic experimental models [9]. In addition to experimental data, large studies have shown an important correlation between diabetes and the incidence of atrial fibrillation.

In Framingham Heart Study, diabetes mellitus was an important prognostic factor for a new odds ratio (OR) for women being 1.6 and 1.4 for men, respectively [10]. In Manitoba Follow-up Study, the prognostic factors for atrial fibrillation were determined in 3983 patients and diabetes mellitus was significantly correlated with atrial fibrillation (relative risk, RR $=1.82$ ) in the mono-factorial analysis [11]. However, in the multi-factorial analysis, this correlation was not statistically significant, arguing that diabetes is not independent prognostic factor for atrial fibrillation. Diabetes mellitus probably promotes the incidence of atrial fibrillation through other risk factors that co-exist in these patients, such as:

- Ischemic heart disease,

- Hypertension and

- Heart failure.

Similarly, the results of a sub-study of the Framingham Heart Study identified independent risk factors for the incidence of atrial fibrillation in 4764 subjects in 10 years of follow-up [12]. It was observed that the association between diabetes mellitus and the incidence of atrial fibrillation did not show a statistically significant ratio $(\mathrm{HR})=1.10,95 \%$ confidence interval $(\mathrm{CI})$ $0.57-1.38, \mathrm{p}=0.43[12]$.

\section{REFERENCES}

[1] Leutholtz BC, Ripoll, I. Diabetes. Exercise and disease management. 2nd ed. Boca Raton: CRC Press 2011.

[2] Vijan S. In the clinic.Type 2 diabetes. Ann Intern Med 2010; 152(5): ITC31-5. http://dx.doi.org/10.7326/0003-4819-152-5-201003020-01003 PMID: 20194231

[3] Fasanmade OA, Odeniyi IA, Ogbera AO. Diabetic ketoacidosis: Diagnosis and management. Afr J Med Med Sci 2008 ; $37(2)$ : 99-105. PMID: 18939392

[4] Pasquel FJ, Umpierrez GE. Hyperosmolar hyperglycemic state: A historic review of the clinical presentation, diagnosis, and treatment. Diabetes Care 2014; 37(11): 3124-31.

http://dx.doi.org/ 10.2337/dc14-0984 PMID: 25342831

[5] Nesto RW, Libby P. Diabetes mellitus and the cardiovascular system. In: Braunwald E, Zipes DP, Libby P, Eds. Heart Disease. A textbook of cardiovascular Medicine. 6th ed. Philadelphia, WB Saunders 2001.

[6] Malmberg K, Yusuf S, Gerstein HC, et al. Impact of diabetes on long-term prognosis in patients with unstable angina and non-Q-wave myocardial infarction: Results of the OASIS (Organization to Assess Strategies for Ischemic Syndromes) Registry. Circulation 2000; 102: 1014-9. http://dx.doi.org/ 10.1161/01.cir.102.9.1014 PMID: 10961966

[7] Fox CS, Coady S, Sorlie PD, et al. Trends in cardiovascular complications of diabetes. JAMA 2004; $292: 2495-9$. http://dx.doi.org/ 10.1001/jama.292.20.2495 PMID: 15562129

[8] Lee CD, Folsom AR, Pankow JS, et al. Cardiovascular events in diabetic and non-diabetic adults with or without history of myocardial infarction. Circulation 2004; 109: 855-60. https://doi.org/10.1161/01.CIR.0000116389.61864.DE

[9] Nichols GA, Reinier K, Chugh SS, et al. Independent contribution of diabetes to increased prevalence and incidence of atrial fibrillation. Diabetes Care 2009; 32: 1851-6.

http://dx.doi.org/ 10.2337/dc09-0939 PMID: 19794003

[10] Benjamin EJ, Levy D, Vaziri SM, D'Agostino RB, Belanger AJ, Wolf PA. Independent risk factors for atrial fibrillation in a population-based cohort. The Framingham Heart Study. JAMA 1994; 271: 840-4. PMID: 8114238

[11] Krahn AD, Manfreda J, Tate RB, et al. The natural history of atrial fibrillation: Incidence, risk factors, and prognosis in the Manitoba Follow-Up Study. Am J Med 1995; 98: 476-84. http://dx.doi.org/ 10.1016/S0002-9343(99)80348-9 PMID: 7733127

[12] Schnabel RB, Sullivan LM, Levy D, et al. Development of a risk score for atrial fibrillation (Framingham Heart Study): A community-based cohort study. Lancet 2009; 373: 739-45.

http://dx.doi.org/ 10.1016/S0140-6736(09)60443-8 PMID: 19249635 